\title{
Performance of Tuned Mass Damper/Double Skin Façade Damping Interaction Systems
}

\author{
K. Moon \\ School of Architecture, Yale University, New Haven, CT 06511, USA
}

\begin{abstract}
Tuned mass dampers (TMDs) can provide very effective solutions for wind-induced vibrations of tall buildings. However, TMDs are usually very large and located near the top of tall buildings for their effective performance. As a result, very valuable occupiable space near the top of tall buildings is sacrificed to contain large TMDs. In addition, installing TMD systems means adding additional masses to tall buildings. As the uses of double skin façade (DSF) systems are increased for tall buildings, this paper investigates an integrated damping system using both TMDs and DSF system that is designed to interact with the TMDs to reduce TMD-related design issues. Compared to the conventional TMD system, the TMD/DSF interaction system requires a significantly reduced TMD mass ratio to achieve the same target damping ratio.
\end{abstract}

\section{Introduction}

As today's tall buildings become ever taller and more slender, wind-induced vibration is a serious design issue. Among various alternative approaches, installing auxiliary damping devices, such as TMDs, is a very reliable solution to mitigate wind-induced vibration of tall buildings. The TMD system is usually composed of one or two large TMD masses located near the top for better performance. As a result, very valuable occupiable space near the top of tall buildings is sacrificed to contain large TMDs.

In order to resolve this issue, vertically distributing multiple smaller TMDs has been investigated by some limited number of researchers such as Bergman et al. (1989) and Moon (2010). In addition, horizontal distribution of multiple TMDs has also been studied by many researchers, such as Kareem and Klein (1995) and Yamaguchi and Harnpornchai (1993).

Regardless of the different distribution strategies, however, installing TMD systems means adding additional masses to tall buildings. In order to address this issue, this paper investigates utilizing existing masses in buildings for the purpose of damping through systems integration. As the uses of double skin façade (DSF) systems are increased for tall buildings, this paper investigates an integrated damping system using TMDs and DSF systems.

The DSF system is composed of two layers of façade. Considering the fact that wind loads are applied to the outer layer of the DSF system first and transmitted to the primary structure of the building, the connectors between the inner and outer layers are designed to provide damping mechanism. When this DSF damping (DSFD) mechanism is applied tall buildings already with TMDs, interactions between the two damping systems may provide synergistic performance characteristics. This paper investigates the performance of TMD/DSFD interaction system. 


\section{Conventional TMDs}

In order to evaluate the structural performance and efficiency of the TMD/DSFD interaction system, the performance of the conventional TMD system is briefly reviewed as a basis of comparison. Figure 1 shows a simplified conventional TMD system model. The system is composed of the primary mass $(\mathrm{M})$, which corresponds to the primary building structure, and the TMD $\left(\mathrm{M}_{\mathrm{d} 1}\right)$. Sinusoidal load, which creates a condition very close to that of organized vortex-shedding, is applied to the primary structure (M). In general, vortex-shedding-induced lock-in phenomena produce the most severe vibration problems for tall buildings (Ali and Moon, 2007; Simiu and Scanlan, 1996).

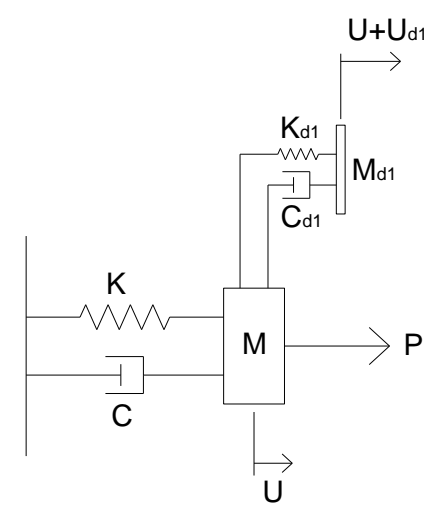

Fig. 1. Simplified conventional TMD system model.

Performance of the conventional TMD system is primarily determined by its mass ratio (Hartog, 1956; Connor, 2003). Figure 2 and 3 show the maximum dynamic amplification factor of the primary structure $(\mathrm{H})$ and TMD mass $\left(\mathrm{H}_{\mathrm{d} 1}\right)$ with optimally tuned TMDs of various mass ratios ranging from $0.1 \%$ to $10 \%$. The inherent damping ratio of the primary building structure is assumed to be $1 \%$.

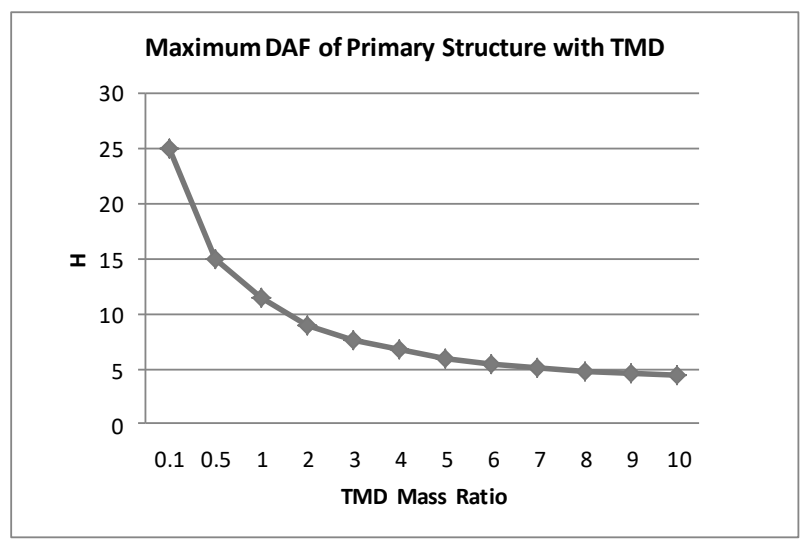

Fig. 2. Maximum dynamic amplification factor of Primary Structure, $H$. 


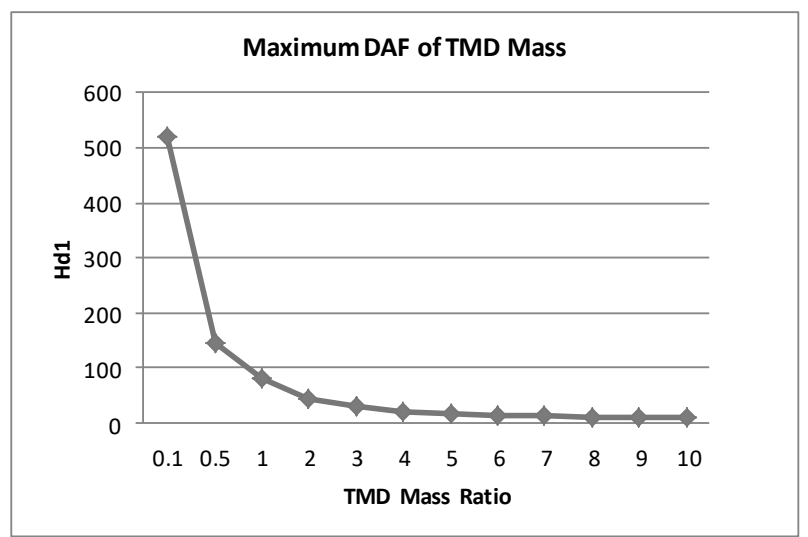

Fig. 3. Maximum dynamic amplification factor of TMD mass, $H_{d 1}$.

\section{TMD/DSFD Interaction Systems}

Before studying the performance of TMD/DSFD interaction systems, a simplified DSFD system model is briefly discussed separately first. As shown in Figure 4, the DSFD system is composed of the primary mass (M), which corresponds to the primary building structure including the inner skin of the DSF system, and the outer skin of the DSF system $\left(\mathrm{M}_{\mathrm{d} 2}\right)$. This model looks, in a sense, similar to the conventional TMD model shown in Figure 1. However, in the TMD model, dynamic force is applied to the primary mass, not to the secondary mass, and the connection between the two masses is tuned to make the TMD mass frequency similar to the primary mass frequency for optimal performance. This type of tuning can make the secondary mass perform as a force amplifier in the DSFD system, the performance of which largely relies on low axial stiffness connectors between the masses.

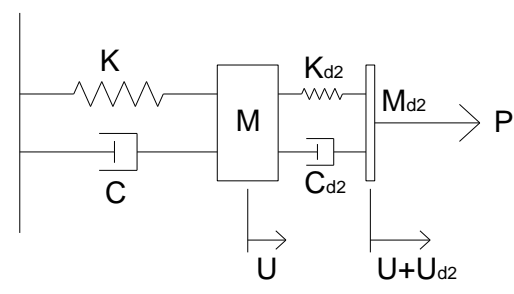

Fig. 4. Simplified DSFD system model.

Figure 5 finally shows a simplified TMD/DSFD interaction system model. The system is now composed of the primary structure (M) including the inner skin of the DSF system, TMD $\left(\mathrm{M}_{\mathrm{d} 1}\right)$ and the DSF outer skin $\left(\mathrm{M}_{\mathrm{d} 2}\right)$. Sinusoidal load, which represents simplified dynamic wind load, is applied to the DSF outer skin $\left(\mathrm{M}_{\mathrm{d} 2}\right)$ in order to anticipate the system performance. 

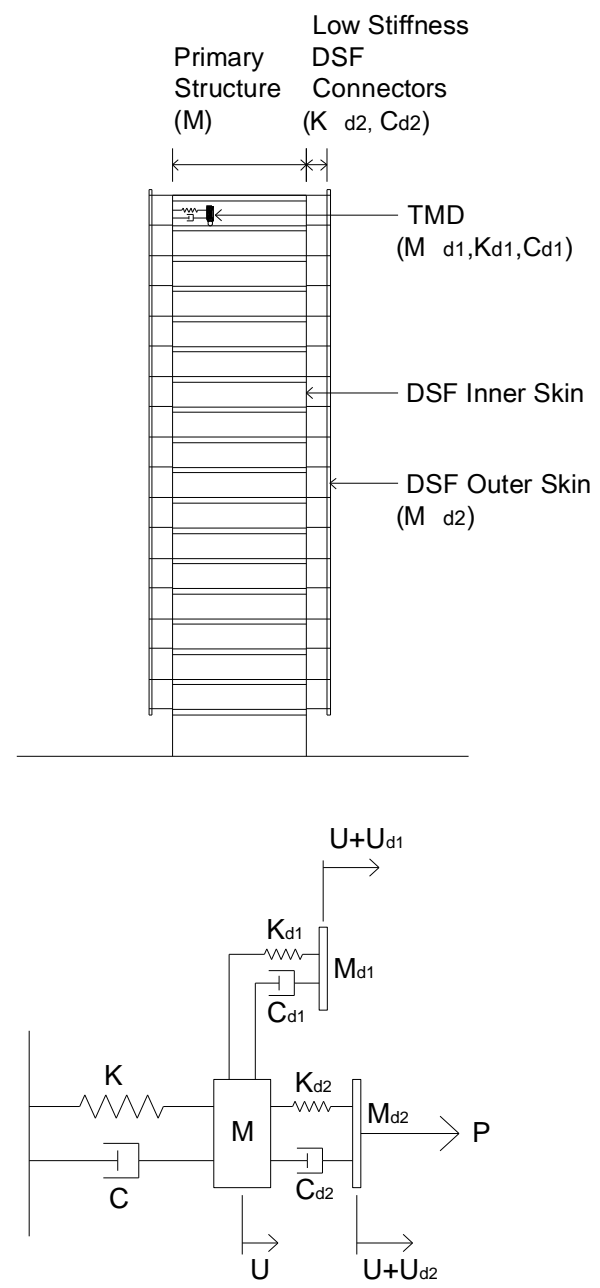

Fig. 5. Simplified TMD/DSFD interaction system model.

The performance of the interaction system is evaluated with the maximum dynamic amplification factor of the primary structure $(\mathrm{H})$, TMD mass $\left(\mathrm{H}_{\mathrm{d} 1}\right)$ and DSF outer skin mass $\left(\mathrm{H}_{\mathrm{d} 2}\right)$. TMD mass ratio is limited to only $0.1 \%$ to estimate the contribution of the DSFD to the interaction system. The inherent damping ratio of the primary building structure is still assumed to be $1 \%$. Without the contribution of the interacting DSFD, the maximum $H$ value of the TMD system is about 25 . This means by installing a TMD system with a mass ratio of $0.1 \%$, the equivalent damping ratio is increased to $2 \%$ by optimal tuning.

This study is performed for tall buildings with DSF systems and the existing DSF outer skin mass ratio is assumed to be $1 \%$. The performance of the interaction system is significantly governed by DSF outer skin to primary structure frequency ratio $\left(\mathrm{f}_{2}\right)$. Figure 6 shows two $\mathrm{H}$ plots with the frequency ratios of 0.5 and 0.25 . With these frequency ratios, the maximum $\mathrm{H}$ values are about 8 and 3, respectively. For optimal performances, DSF connector damping ratios of 0.08 and 0.2 are also used respectively. When the 
conventional TMD system is used, the same $\mathrm{H}$ values can be achieved with the TMD mass ratio of about $2 \%$ and well over $10 \%$, respectively. Note that in this study the TMD mass ratio is only $0.1 \%$ and the existing DSF outer skin mass ratio is assumed to be $1 \%$.
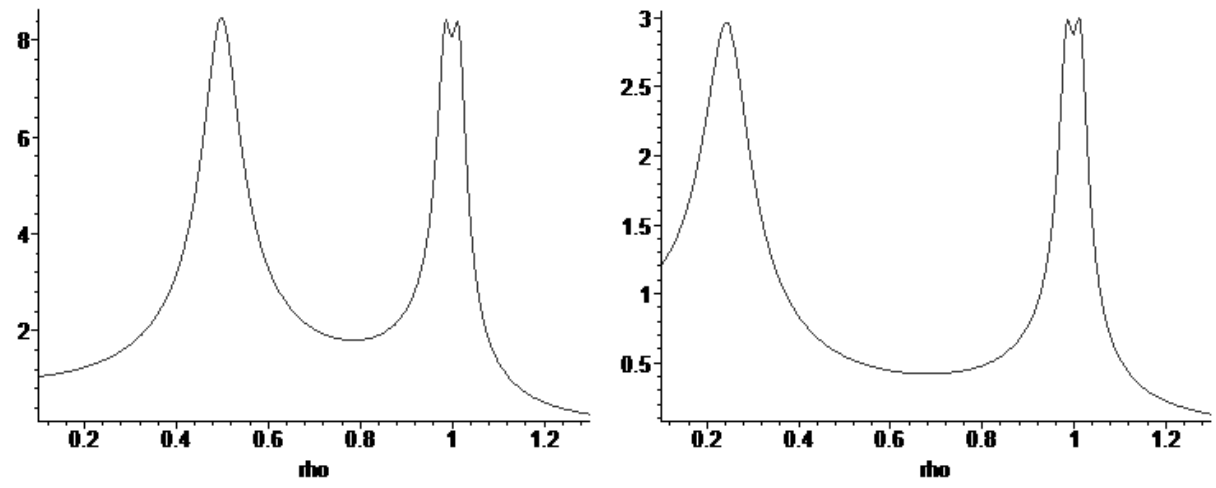

Fig. 6. $\mathrm{H}$ plots of TMD/DSFD system with $\mathrm{f}_{2}$ values of 0.5 (left) and 0.25 (right) [rho: forcing frequency to natural frequency of the building ratio].

While the vibration of the primary structures can be reduced much more efficiently with the TMD/DSFD interaction system, excessive movements of the DSF outer skin mass are a serious design issue of the interaction system. Figure 7 shows $\mathrm{H}_{\mathrm{d} 2}$ plots corresponding to $\mathrm{H}$ plots shown in Figure 6. As can be seen from the plots as $\mathrm{f}_{2}$ value is decreased maximum $\mathrm{H}_{\mathrm{d} 2}$ values increase significantly. Increasing DSF outer skin mass ratios and connector damping ratios can substantially contribute to controlling the excessive movements of the DSF outer skin masses. However, DSF outer skin mass ratios larger than about $1 \%$ may be impractical, and DSF connector damping ratios larger than the optimal values result in increased vibration of the primary structure as well as the TMD mass. Therefore, design parameters should be carefully determined to control vibrations of the primary structure, TMD masses and DSF outer skin masses.
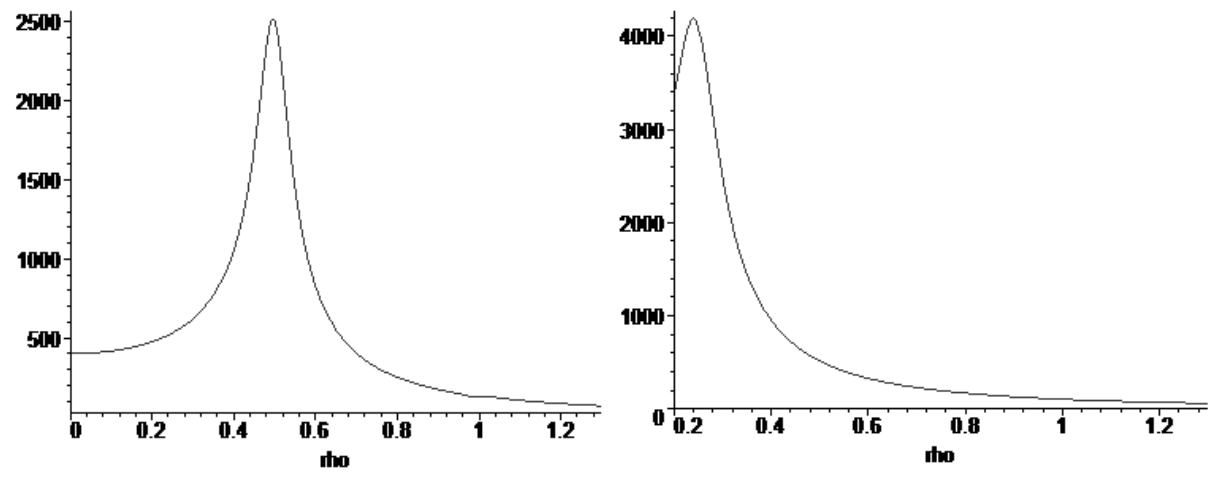

Fig. 7. $\mathrm{H}_{\mathrm{d} 2}$ plots of TMD/DSFD system with $\mathrm{f}_{2}$ values of 0.5 (left) and 0.25 (right) [rho: forcing frequency to natural frequency of the building ratio]. 


\section{Conclusions}

An integrative design strategy to mitigate wind-induced vibration of tall buildings has been presented through studies on the TMD/DSFD interaction system. Compared to the conventional TMD system, the TMD/DSFD interaction system requires a significantly reduced TMD mass ratio to achieve the same target damping ratio. However, excessive vibrations of the DSF outer skin masses are a serious design limitation. Though the primary purpose of the damping system is to reduce the vibrations of the primary structure, design parameters should be carefully determined to control not only vibrations of the primary structure but also excessive movements of the TMD masses and DSF outer skin masses. Further studies are required for practical applications of the presented theoretical studies. With rapid development of new building technologies, studies on more integrative ways of designing building systems are crucial to synergistically enhance their performances and eventually produce higher quality built environments.

\section{References}

Ali, MM. and Moon K. 2007. Structural Developments in Tall Buildings: Current Trends and Future Prospects. Architectural Science Review, Vol. 50.3, pp 205223.

Bergman LA, McFarland DM, Hall JK, Johnson EA, Kareem A. 1989. Optimal distribution of tuned mass dampers in wind-sensitive structures. Structural Safety and Reliability: Proceedings of ICOSSAR '89, the $5^{\text {th }}$ International Conference on Structural Safety and Reliability, ASME, New York, 95-102.

Connor JJ. 2003. Introduction to Structural Motion Control. Prentice Hall: New York. Hartog D. 1956. Mechanical Vibrations, 4th Ed. McGraw-Hill: New York.

Kareem, A. and Klein, S. 1995. Performance of Multiple Mass Dampers Under Random Loading. Journal of Structural Engineering, Vol.121.2, pp 348-361, 1995.

Moon K. 2010. Vertically Distributed Multiple Tuned Mass Dampers in Tall Buildings: Performance Analysis and Preliminary Design, The Structural Design of Tall and Special Buildings, Vol. 19, 347-366.

Moon K. 2009. Tall Building Motion Control Using Double Skin Facades, ASCE Journal of Architectural Engineering, Vol. 15-3, 84-90.

Simiu, E. and Scanlan, RH. 1996. Wind Effects on Structures: Fundamentals and Applications to Design. $3^{\text {rd }}$ Edition. New York: Wiley

Smith RJ, Willford MR. 2007. The Damped Outrigger Concept for Tall Buildings, The Structural Design of Tall and Special Buildings, Vol.19-4, 501-517.

Oesterle E, Lieb R, Lutz M, Heusler W. 2001. Double-Skin Facades, Munich: Prestel.

Yamaguchi, H. and Harnpornchai N. 1993. Fundamental Characteristics of Multiple Tuned Mass Dampers for Suppressing Harmonically Forced Oscillation. Earthquake Engineering and Structural Dynamics, Vol.22, pp 51-62. 